\title{
When the Perfect Environment is Not Enough for People Living with Dementia
}

\author{
Dawn Wiggins* \\ University of Stirling, Canada
}

*Corresponding author: Dawn Wiggins, University of Stirling, Canada

\begin{abstract}
This opinion piece will discuss the differences between treating dementia the disease and treating the person who happens to have dementia. When one treats the person rather than the disease, there is a better chance of collaboration and understanding and greater opportunity for both the carer and the person with dementia to feel seen and understood and to have a good experience. One major element of focus in regard to treating the person rather than the disease with the use of humor and laughter. Proper training for the family member and carer will be a smaller focus in this opinion piece. Finally, the author's professional experience in the field of dementia care will be highlighted in order to strengthen the ideas and theory being presented.
\end{abstract}

Keywords: Dementia; Carer; Humor; Healing; Care partnerships; Dementia education; Self-care; Memory care

\section{Introduction}

As Patch Adams, MD once said, "You treat a disease, you win, you lose. You treat a person I'll guarantee you'll will win no matter the outcome." The same can be said about people living with dementia. If you treat the disease, you're going to eventually lose. But if you treat the person-including co-creating care and treatment plans through authentic partnerships-you are guaranteed to win. One manner of creating a care partnership is to intentionally build and create a living environment that is designed to cater to the needs of people living with dementia. These kinds of facilities are being built all over the world, but simply designing and creating them is still not enough. When establishing care partnerships, we need to build an environment around vulnerability, the carer included, and around true human connection. Through education we must encourage carers to see people for who they really are and utilize one of nature's most beneficial and healing remedies-laughter.

\section{Discussion}

I recently created the Dr. Sandra Black Centre for Memory Care (Name has been changed) in a retirement community in Ontario, Canada. It was built for the sole purpose of creating a warm environment for people living with dementia and each room was designed to create meaningful engagement between residents, staff, and family members. There was a beach room, a barber shop, a nursery, and a coffee shop-all put into the centre intentionally to provide meaning while living with dementia. From a personal standpoint as well as a cultural standpoint, each of these rooms have a different and unique significance. The Black Centre had great promise and was quite beneficial to the community; not just staff, residents, and families but also people who lived near the centre. I stopped working there for a year and when I came back the staff had all of the residents sitting in front of a TV, unengaged and deteriorating, instead of engaging in the various specialized rooms and what was set up to be a warm, welcoming and purposeful environment.

It was then that I realized that when the centre was created, it was not done so in collaboration and partnership with persons living with dementia in the facility. What we needed was training for the staff which would then allow the staff and the residents to come together to create a welcoming and more engaging environment. The connection and relationship one has with the person living with dementia is important. It is crucial that the staff see the person with dementia as a human being and not just a diagnosis. It's also necessary that the staff member make themselves aware of the changes the person living with dementia is going through-vision changes, language changes, taste changes, and sensory motor changes-and how these changes uniquely present themselves in 
that person. One must learn how to interact with and support the person living with dementia in a way that honors their dignity and personhood. Instead of trying to correct the person with dementia, we needed to retrain staff to try to validate the experience of the person they are caring for and operate from a strengths-based point of view ("you're trying so hard and doing the best you can," rather than, "why can't you ever finish your sentences"). We had to work with new staff to let them know that if the person with dementia became angry with them, they needed to try not to take it personally because the person was not aware of what they were doing. The person with dementia lacks the ability to be reasonable and rational and has a hard time making choices and has a decrease in their impulse control.

One major element we built into our training that we needed to become part of the everyday environment-humor. The Association for Applied and Therapeutic Humor states, "humor provides innumerable benefits to our overall health and well-being, including reduced stress, greater resilience, decreased depressive symptoms, and even increased pain tolerance."

Because of stigma and shame due to misinformation, often a person living with dementia isolate, which one can do alone or even in a crowded room. The effects of isolation for a person with dementia include depression, anxiety, and even suicide ideation and attempts. The carer or care partner has a difficult role; sometimes giving of themselves with emotional support and quite often a physical support. But who cares for the care partner? One cannot constantly fill someone else's cup without replenishing their own. Without adequate self-care, the carer can fall into depression and anxiety or other serious health issues like cardiovascular disease or addictions.

At the Dr. Sandra Black Centre for Memory Care, humor has shown to be a tremendous coping mechanism and skill for the carer and for the person living with dementia. The staff members have shown to practice more self-care and have higher engagement in their day to day activities and more positive interactions with each other and with the residents. Humor has helped the residents willingly participate in their own care and treatment in a meaningful way and has shown to help increase levels of fulfillment as well.

Integrating humor into our training and making it a part of everyday life has helped turn conflict between residents and between residents and staff into teachable moments and friendships.

\section{Conclusion}

Disputes and difficulties are bound to arise between human beings. Throw in neurocognitive decline into the mix and you have a recipe for misery, despair, and battles fought with sharp words and harmful actions fueled by hurt emotions. But when there is a commitment to learning, both the carer (the professional or the layperson) and the person with dementia will then grow and become more vulnerable. From that vulnerable place, humor is born, which can help diffuse tension, smooth over misunderstandings, and provide much needed perspective when life becomes seemingly unbearable or difficult. It is in this place where collaborative treatment is given priority, and a space intentionally created for persons living with dementia can have the most meaning and impact. Only then can both the carer and the person with dementia celebrate a win because it is the person and not the disease that is being treated.

\section{Acknowledgement}

None.

\section{Conflict of Interest}

No conflict of interest. 